\title{
TWENTY-TWO PRIMES IN ARITHMETIC PROGRESSION
}

\author{
PAUL A. PRITCHARD, ANDREW MORAN, AND ANTHONY THYSSEN
}

ABSTRACT. Some newly-discovered arithmetic progressions of primes are presented, including five of length twenty-one and one of length twenty-two.

Grosswald [1] strengthened a special case of a famous conjecture of Hardy and Littlewood to give an asymptotic estimate of the number of arithmetic progressions of primes (PAPs) of length $m$ with no term exceeding $x$. Unless its first term is $m$, a PAP of length $m$ must have a common difference divisible by $\Pi(m)$, the product of primes $\leq m$. Pritchard [6] noted that Grosswald's formula could be generalized to estimate the number of PAPs of length $m$ with common difference divisible by $\Pi(n)$ and no term exceeding $x$ (for $m \leq n$ ).

Pritchard [6] reported on an extensive search for "long" PAPs, which produced results consistent with the generalized formula of Grosswald, and which found the then longest known PAP, of length 19. We report herein on what has been discovered since.

As reported by Guy [3], James Fry and Jeff Young found three PAPs of length 20 in 1987. We have performed four searches since.

Our first two searches were distributed over about 40 Sun/3 workstations in the Department of Computer Science at the University of Queensland. The software used is described in [4]. The crucial search algorithm improves on that described in [5] by exploiting bit-parallel techniques.

The first search revealed that one of the PAPs found by Fry and Young was the PAP of length 20 with minimum last term:

$$
214861583621+18846497670 k, \quad k=0,1, \ldots, 19 .
$$

Its common difference is $1943 \times \Pi(19)$. (Here and below we report the common difference in the form $f \times \Pi(n)$ where $n$ is prime and maximal.)

The second search found the first known PAP of length 21 in 1990:

$$
142072321123+1419763024680 k, \quad k=0,1, \ldots, 20 .
$$

Its common difference is $6364 \times \Pi(23)$.

Our third search, using a more sophisticated method of distribution designed by the first and third authors, was distributed over about 20 (eventually) Sun SPARCstations, at Griffith University in Brisbane, Australia, and the University of Bergen in Norway. It found two more PAPs of length 21:

$$
5749146449311+26004868890 k, \quad k=0,1, \ldots, 20,
$$

Received by the editor September 5, 1992 and, in revised form, August 2, 1994.

1991 Mathematics Subject Classification. Primary 11N13, 11 Y11. 
TABLE 1. PAPs (with common difference $f \times \Pi(n)$ ) found by third search

\begin{tabular}{|c|r|r|c|}
\hline length & \multicolumn{1}{|c|}{ first term } & \multicolumn{1}{|c|}{$f$} & $n$ \\
\hline 20 & 68469367129 & 4926 & 19 \\
20 & 109405841773 & 29684 & 19 \\
20 & 214861583621 & 1943 & 19 \\
20 & 474054896773 & 6321 & 19 \\
20 & 803467381001 & 9 & 23 \\
20 & 882050255881 & 10525 & 19 \\
20 & 1140997291211 & 788 & 19 \\
20 & 1361328929537 & 19511 & 19 \\
20 & 2364458499701 & 11583 & 19 \\
20 & 2750642120531 & 4663 & 19 \\
20 & 2773814832407 & 155 & 19 \\
20 & 3313355036261 & 10878 & 19 \\
20 & 3362427181159 & 5956 & 19 \\
20 & 3774669829057 & 3205 & 19 \\
20 & 4251333799021 & 7833 & 19 \\
20 & 4252782701327 & 2470 & 19 \\
21 & 5749146449311 & 2681 & 19 \\
\hline
\end{tabular}

TABLE 2. Actual versus estimated numbers of PAPs

\begin{tabular}{|c|c|c|}
\hline \multirow{2}{*}{ length } & \multicolumn{2}{|c|}{ Number of PAPs } \\
\cline { 2 - 3 } & actual & estimated \\
\hline 20 & 18 & 13 \\
21 & 1 & 1.4 \\
\hline
\end{tabular}

with a common difference of $2681 \times \Pi(19)$, and

$$
2930617401661+285801365850 k, \quad k=0,1, \ldots, 20,
$$

with a common difference of $29465 \times \Pi(19)$. The former is the PAP of length 21 with minimum last term.

This third search can be used to test Grosswald's estimate, since it found all PAPs of length at least 20 with no term exceeding $646433 \times \Pi(19)=$ 6270199705770 . (It actually started with a bigger limit which was reduced when each of the 2 PAPs of length 21 were found.) The complete list of the PAPs found in the above range is given in Table 1.

Table 2 shows the actual numbers of PAPs found (in the above range) versus the numbers estimated using the first two terms of the generalized formula of Grosswald (an infinite series).

Our fourth search was designed to find a PAP of length 22, and was limited to PAPs with common difference divisible by $\Pi(23)$ in order to speed up the search. It found two more PAPs of length 21:

$$
14676404481107+2232044164350 k, \quad k=0,1, \ldots, 20 \text {, }
$$


with a common difference of $345 \times \Pi(29)$, and

$$
28383220937263+1861263814410 k, \quad k=0,1, \ldots, 20,
$$

with a common difference of $8343 \times \Pi(23)$.

Finally, in March 1993, it found the first known PAP of length 22:

$$
11410337850553+4609098694200 k, \quad k=0,1, \ldots, 21,
$$

with a common difference of $20660 \times \Pi(23)$. It is extremely unlikely that this is the PAP of length 22 with minimum last term. That awaits another search.

\section{ACKNOWLEDGMENTS}

Thanks to all the tolerant users and helpful system administrators at the School of Computing and Information Technology at Griffith University, the Department of Informatics at the University of Bergen, and the Department of Computer Science at the University of Queensland.

\section{BIBLIOGRAPHY}

1. E. Grosswald, Arithmetic progressions that consist only of primes, J. Number Theory 14 (1982), 9-31.

2. R. K. Guy, Unsolved problems in number theory, Springer-Verlag, New York, 1981.

3. __ Canadian number theory association unsolved problems 1988, Number Theory (R. A. Mollin, ed.), de Gruyter, Berlin, 1990, pp. 193-206.

4. A. Moran and P. A. Pritchard, The design of a background job on a local-area network, Proceedings 14th Australian Computer Science Conference (G. Gupta, ed.), Australian Computer Science Communications 13 (1991), 17-1-17-11.

5. P. A. Pritchard, A case study of number-theoretic computation: searching for primes in arithmetic progression, Sci. Comput. Programming 3 (1983), 37-63.

6. $\ldots$, Long arithmetic progressions of primes: some old, some new, Math. Comp. 45 (1985), 263-267.

School of Computing and Information Technology, Griffith University, QueEnsLAND, Australia 4111

E-mail address: p.pritchard@cit.gu.edu.au

Department of Computing Sciences, Chalmers University of Technology, S-412 96, GÖTEBORG, SWEDEN

E-mail address: andrew@cs. chalmers.se

School of Computing and Information Technology, Griffith University, QueensLAND, Australia 4111

E-mail address: a.thyssen@cit.gu.edu.au 